\title{
ON RINGS WITH INVARIANT RADICALS
}

\begin{abstract}
A.V. Kelarev and A. Plant
We give necessary and sufficient conditions on the semigroup $S$ for the Jacobson radical to be $S$-invariant.
\end{abstract}

Our main theorem is motivated by earlier results on the radicals of group graded rings, Morita contexts, and generalised matrix rings. All of these rings can be viewed as semigroup graded rings.

Let $S$ be a semigroup. A ring $R$ is said to be $S$-graded if $R=\bigoplus_{s \in S} R_{s}$ is a direct sum of additive subgroups $R_{s}$ and $R_{s} R_{t} \subseteq R_{s t}$ for all $s, t \in S$. Let $B_{n}$ be the semigroup consisting of zero and all the standard $n \times n$ matrix units. Morita contexts are $B_{2}$-graded and generalised matrix rings are $B_{n}$-graded $[1,12]$.

Several authors have considered radicals invariant in group graded rings and Morita contexts $[1,4,5,11]$. We give a definition which unifies these two cases.

The Jacobson radical of $R$ is denoted by $\mathcal{J}(R)$. For any semigroup $S$, let

$$
S^{*}= \begin{cases}S \backslash\{0\} & \text { if } S \text { has a zero } \\ S & \text { otherwise. }\end{cases}
$$

We say that the Jacobson radical is $S$-invariant if and only if $R_{x} \mathcal{J}\left(R_{e}\right) R_{y} \subseteq \mathcal{J}\left(R_{f}\right)$ for every $S$-graded ring $R=\bigoplus_{\in \in S} R_{a}$, where $e, f$ are any nonzero idempotents of $S$ and $x, y$ are any elements of $S$ such that $x e y=f$.

It is known that the Jacobson radical is $S$-invariant if $S$ is a group or $S=B_{n}$ [2, $7,12]$. The aim of this paper is to describe all semigroups $S$ such that the concept of invariant radicals can be used in $S$-graded rings. The answer is given in terms of inverse semigroups and primitive idempotents. Inverse semigroups form an important class arising in many interesting settings. For the previous results on inverse semigroups we refer the reader to [10]. A semigroup $S$ is said to be inverse if for every $s \in S$ there exists a unique $t \in S$ such that $s t s=s$ and $t s t=t$. Inverse semigroup algebras give examples of rings graded by inverese semigroups [9]. Rings graded by inverse semigroups with finitely many idempotents were considered in [7].

Received 11th April, 1995

Copyright Clearance Centre, Inc. Serial-fee code: 0004-9729/96 \$A2.00+0.00. 
If $e, f$ are idempotents of a semigroup $S$, we shall write $e \leqslant f$ if $e f=f e=e$. It is easily verified that $\leqslant$ is a partial order relation on the set $E(S)$ of idempotents of $S$. An idempotent is called primitive if it is nonzero and is minimal in the set of nonzero idempotents (with respect to the order just described).

THEOREM 1. Let $S$ be a semigroup, $L(S)$ the union of all ideals of $S$ which do not contain nonzero idempotents, and let $P(S)$ be the ideal generated by all idempotents in $S$. Then the following conditions are equivalent:

(i) the Jacobson radical is $S$-invariant;

(ii) the quotient semigroup $P(S) / L(S)$ is an inverse semigroup in which every nonzero idempotent is primitive.

The following definitions, examples and lemmas are required for the proof. A semigroup of idempotents is called a band. If a band satisfies the identity $x y=x$ $(x y=y)$, then it is called a left zero band (right zero band). A commutative band is called a semilattice.

Denote by $e_{i j}$ the standard matrix unit with the identity in the $i, j$ entry and all other entries zero.

Lemma 2. Let $S$ be a semigroup and $e, f \in S^{*}$ be idempotents such that $S e S \supset$ $S f S$. Then there exists an $S$-graded ring $R$ such that the Jacobson radical is not $S$-invariant.

Proof: Let $U=S e S$ and $V=S f S$. Since $f \in U$, then there exist $x, y \in S$ such that $f=x e y$. We may assume that $x$ and $y$ belong to $V$ because otherwise we could replace $x$ and $y$ by $f x \in V$ and $y f \in V$ using the equality $f=f^{3}=(f x) e(y f)$. Let $M=\mathbb{R}_{2}$, the ring of $2 \times 2$ matrices with entries from the field of reals $\mathbb{R}$, and let $T$ be the subring given by $e_{12} \mathbb{R}$. Consider the semigroup ring $M S$. Clearly $M V$ is an ideal of $M S$ and $T U$ is a subring of $M S$. Hence the sum $R=T U+M V$ is a subring of $M S$. For any $s \in S$ we put

$$
R_{s}= \begin{cases}M s & \text { if } s \in V \\ T s & \text { if } s \in U \backslash V \\ 0 & \text { if } s \notin U .\end{cases}
$$

Then $R_{x}=M_{x}, R_{y}=M_{y}, R_{f}=M f$ and $R_{e}=T_{e}$. Since $R_{e}^{2}=T^{2} e^{2}=0$ it follows that $R_{e}$ is quasiregular. Thus $0 \neq e_{12} f=\left(e_{11} e_{12} e_{22}\right)(x e y)=\left(e_{11} x\right)\left(e_{12} e\right)\left(e_{22} y\right) \in$ $R_{x} \mathcal{J}\left(R_{e}\right) R_{y}$. It follows that the Jacobson radical is not invariant, because obviously $\mathcal{J}\left(R_{f}\right)=0$ cannot contain $e_{12} f$.

Lеммa 3. Let $S$ be a semigroup. If $S$ contains a subsemigroup isomorphic to the two-element left zero band $X_{2}$ (or the two-element right zero band $Z_{2}$ ), then there exists an $S$-graded ring $R$ such that $\mathcal{J}(R)$ is not invariant. 
PROOF: We shall consider only the case when $S$ contains a subsemigroup isomorphic to the two element left zero band, as the case when $S$ contains a subsemigroup isomorphic to the two-element right zero band is similar.

Let $M=\mathbb{R}_{2}$, the ring of $2 \times 2$ matrices with entries from the field of reals $\mathbb{R}$, and consider its right ideals

$$
\begin{aligned}
& M_{x}=\left\{\left[\begin{array}{ll}
a & b \\
0 & 0
\end{array}\right] \mid a, b \in \mathbb{R}\right\} \\
& M_{y}=\left\{\left[\begin{array}{ll}
0 & 0 \\
c & d
\end{array}\right] \mid c, d \in \mathbb{R}\right\} .
\end{aligned}
$$

If we consider $X_{2}=\{x, y\}$ as a left zero band, then $M=M_{x}+M_{y}$ is $X_{2}$-graded. Clearly $I_{x}=\left\{r e_{12} \mid r \in \mathbb{R}\right\}$ is an ideal of $M_{x}$. It follows that $\mathcal{J}\left(M_{x}\right)=I_{x}$. Similarly $\mathcal{J}\left(M_{y}\right)=\left\{r e_{21} \mid r \in \mathbb{R}\right\}$.

Now, suppose that the Jacobson radical is invariant. Then

$$
e_{22}=e_{21} e_{12} e_{22} \in M_{y} \mathcal{J}\left(M_{x}\right) M_{y} \subseteq \mathcal{J}\left(M_{y}\right)
$$

However, $e_{22} \notin \mathcal{J}\left(M_{y}\right)$. This is a contradiction and the Jacobson radical is not invariant.

Proof of the Main Theorem: (i) $\Rightarrow$ (ii) : Suppose that the Jacobson radical is $S$-invariant. Denote by $E(S)$ the set of all idempotents in $S$ and let $P(S)$ be the ideal generated by $E(S)$. If $P(S)=\emptyset$, the assertion is trivial, and so we may assume $P(S) \neq \emptyset$. Let $L(S)$ be the union of all ideals of $S$ which do not contain nonzero idempotents. Clearly $P(S) \neq L(S)$.

We shall use the same letters to denote the elements in $S$ and their images in the quotient semigroup $P(S) / L(S)$.

For any nonzero element $a \in P(S) / L(S)$ denote by id $(a)$ the ideal generated by $a$, by $I(a)$ the ideal of non-generating elements, and by $F_{a}$ the principal factor id $(a) / I(a)$ containing $a$.

First, suppose that $P(S) / L(S)$ has a nonzero idempotent $e$ which is not primitive. Then $P(S) / L(S)$ contains a nonzero idempotent $f \neq e$ such that $e f=f e=f$. Therefore id $(f) \subset$ id $(e)$. This contradicts Lemma 2 and it follows that all nonzero idempotents of $P(S) / L(S)$ are primitive.

Next, take any nonzero $a \in P(S) / L(S)$. We shall show that $a$ has an inverse element. To this end, it suffices to verify that the principal factor $F_{a}$ is inverse.

Since $a \in P(S)$, there exists a nonzero idempotent $e \in E(S)$, with $a \in \operatorname{id}(e)$. Since $a \notin L(S)$, then there exists an idempotent $f \in \operatorname{id}(a)$. So id $(f) \subseteq \operatorname{id}(a) \subseteq \operatorname{id}(e)$. Lemma 2 shows that $\operatorname{id}(f)=\operatorname{id}(a)=\operatorname{id}(e)$. 
Consider the principal factor $F_{a}$. Since $F_{a}$ contains a primitive idempotent (namely $e$ ), it follows from [3, Lemma 2.39], that $F_{a}$ is completely 0 -simple. Now, by Lemma $3, F_{a}$ cannot contain a subsemigroup isomorphic to the left or right zero band. From [3, Lemma 2.14 and Corollary 2.19], it follows that $F_{a}$ is an inverse semigroup and so $a$ has an inverse element. Since $a$ was an arbitrary element, it follows that $P(S) / L(S)$ is an inverse semigroup in which every nonzero idempotent is primitive.

(ii) $\Rightarrow$ (i) : Let $S$ be a semigroup and let $R$ be an $S$-graded ring. Suppose that the quotient semigroup $P(S) / L(S)$ is an inverse semigroup in which every nonzero idempotent is primitive.

Let $G$ be a group and $I$ a nonempty indexing set. Then the set of triples $\{(i, g, j)\}$ forms a semigroup with multiplication defined by

$$
(i, a, j)(k, b, l)= \begin{cases}(i, a b, l) & \text { if } j=k \\ 0 & \text { if } j \neq k\end{cases}
$$

This semigroup is called the Brandt semigroup. From [3, Exercise 6 of Section 6.5], it follows that $P(S) / L(S)$ is a 0 -direct union of Brandt semigroups.

Let $e, f \in S$ be idempotents and take any $x, y \in S$ such that $e=x f y$. Then the idempotents $e$ and $f$ must belong to the same 0-direct component of $P(S) / L(S)$.

First, consider the case when $x$ and $y$ are also in the same 0 -direct component as $e$ and $f$. Pass to the principal factor $F$ of $P(S) / L(S)$ containing $e, f, x, y$. Again we shall use the same letters $e, f, x, y$ to denote the images of $e, f, x$ and $y$ in $F$.

Let $1 \in G$ denote the identity element of $G$. Since $e, f \notin L(S)$, then clearly $e, f$ are nonzero idempotents of $F$. It is easily seen that $e=(i, 1, i)$ and $f=(j, 1, j)$ for some $i, j \in I$. It follows from the equality $x f y=e$ that if $x=(i, g, j)$ for some $g \in G^{0}$, then $y=\left(j, g^{-1}, i\right)$.

Denote by $\mathcal{R}_{g}$ the sum of all $R_{(i, g, j)}$ where $i, j \in I, g \in G^{0}$. For any $g, h \in G^{0}$ and any $i, j, k, l \in I$, we get $(i, g, j),(k, h, l)=(i, g h, l)$ if $j=k$, and $(i, g, j)(k, h, l)=0$ otherwise. In both cases $R_{(i, g, j)} R_{(h, k, l)} \subseteq \mathcal{R}_{g h}$. Therefore $\mathcal{R}=\bigoplus_{g \in G^{0}} \mathcal{R}_{g}$ is $G^{0}$-graded. The identity component, $\mathcal{R}_{1}=\bigoplus_{i, j} R_{(i, 1, j)}$ is a generalised matrix ring. Therefore $\mathcal{J}\left(\mathcal{R}_{1}\right) \cap R_{e}=\mathcal{J}\left(R_{e}\right)$ for any idempotent $e \in S$ (see [6, Theorem 5.1], together with [8, Proposition 6.18]). Since $\mathcal{R}$ is group graded, $\mathcal{J}(\mathcal{R})$ is $G^{0}$-invariant (see [12] together with [1]). Therefore $\mathcal{R}_{g} \mathcal{J}\left(\mathcal{R}_{1}\right) \mathcal{R}_{g^{-1}} \subseteq \mathcal{J}\left(\mathcal{R}_{1}\right)$. Since $R_{x} \subseteq \mathcal{R}_{g}$ and $R_{y} \subseteq \mathcal{R}_{g^{-1}}$ we get $R_{x} \mathcal{J}\left(R_{f}\right) R_{y} \subseteq \mathcal{R}_{g} \mathcal{J}\left(\mathcal{R}_{1}\right) \mathcal{R}_{g^{-1}} \cap R_{x} R_{f} R_{y} \subseteq \mathcal{J}\left(\mathcal{R}_{1}\right) \cap R_{e}=\mathcal{J}\left(R_{e}\right)$, as required.

Second, consider arbitrary $x, y \in S$. Put $M=R_{x} \mathcal{J}\left(R_{f}\right) R_{y}$ and let $T=R_{e}^{1} M R_{e}^{1}$ be the ideal generated by $M$ in $R_{e}$. In order to prove $M \subseteq \mathcal{J}\left(R_{e}\right)$ we shall show that $T$ is quasiregular.

Let $F$ be the principal factor of $S$ containing $e$ and $f$. Then $e x$ and ye are nonzero 
elements of $F$, because otherwise we would get $e=$ exfye $=0$. For such elements we have proved that $\mathcal{J}\left(R_{e}\right) \supseteq R_{e x} \mathcal{J}\left(R_{f}\right) R_{y e}$. Consider the ideal $T^{3} \subseteq T R_{e}^{1} M R_{e}^{1} T \subseteq$ $R_{e} R_{x} \mathcal{J}\left(R_{f}\right) R_{y} R_{e} \subseteq R_{e x} \mathcal{J}\left(R_{f}\right) R_{y e} \subseteq \mathcal{J}\left(R_{e}\right)$. Hence $T$ is quasiregular, as required. [

\section{REFERENCES}

[1] S.A. Amitsur, 'Rings of quotients and Morita contexts', J. Algebra 17 (1971), 273-298.

[2] G.M. Bergman, 'Radicals, tensor products, and algebraicity', in Ring Theory 1989 in honor of S.A. Amitsur (The Weizmann Science Press of Israel, 1989), pp. 150-192.

[3] A.H. Clifford and G.B. Preston, The algebraic theory of semigroups 1 and 2 (American Mathematical Society, Rhode Island, 1964).

[4] M. Cohen and S. Montgomery, 'Group-graded rings, smash products, and group actions', Trans. Amer. Math. Soc. 282 (1984), 237-258.

[5] M. Jaegermann and A.D. Sands, 'On normal radicals, $N$-radicals, and $A$-radicals', $J$. Algebra 50 (1978), 337-349.

[6] E. Jespers, 'Radicals of graded rings', in Theory of radicals Szekszárd, Hungary (Colloquia Mathematica Societatis János Bolyai 61, 1993), pp. 109-130.

[7] E. Jespers and P. Wauters, 'Rings graded by an inverse semigroup with finitely many idempotents', Houston J. Math. 15 (1989), 291-304.

[8] G. Karpilovsky, The Jacobson radical of classical rings (John Wiley and Sons, New York, 1991).

[10] W.D. Munn, 'Inverse semigroup algebras', in Group and semigroup rings (North-Holland, New York, 1986), pp. 197-223.

[11] M. Petrich, Inverse semigroups (John Wiley and Sons, New York, 1984).

[12] A.D. Sands, 'On normal radicals', J. London Math. Soc. 11 (1975), 361-365.

[13] A.D. Sands, 'On invariant radicals', Canad. Math. Bull. 32 (1989), 255-256.

Department of Mathematics

University of Tasmania

Hobart, Tas. 7001

Australis

e-mail: kelarev@hilbert.maths.utas.edu.au

plant@hilbert.maths.utas.edu.au 